\title{
Cocaine-Associated Stimuli Increase Cocaine Seeking and Activate Accumbens Core Neurons after Abstinence
}

\author{
Jonathan A. Hollander and Regina M. Carelli \\ Department of Psychology, The University of North Carolina at Chapel Hill, Chapel Hill, North Carolina 27599-3270
}

\begin{abstract}
Electrophysiological recordings were completed in rats $(n=14)$ trained to self-administer cocaine to determine whether activation of nucleus accumbens (Acb) neurons (core vs shell) by cocaine-associated stimuli is enhanced after 1 month of cocaine abstinence. After self-administration training, 170 cells were recorded during a single test session conducted either the next day or 1 month later. The test session consisted of three phases during which (1) the cocaine cue was presented unexpectedly to rats, (2) rats responded for the same cue in the absence of the drug (extinction), and (3) the cocaine cue was presented randomly between cocaine-reinforced responding during resumption of self-administration. The cocaine stimulus significantly increased activation of Acb core (not shell) neurons after 1 month of cocaine abstinence (compared with $1 \mathrm{~d}$ ); this finding occurred regardless of contingency of cue presentation or cocaine availability. Acb core activation was not observed in other rats $(n=7)$ presented with the same stimulus never paired with cocaine. The results reflect a cellular neuroadaptation in the Acb core related to cocaine-associated cues that is observed during initial cue exposure and sustained during extinction and resumption of self-administration after prolonged drug abstinence.
\end{abstract}

Key words: electrophysiology; behavior; self-administration; relapse; extinction; withdrawal

\section{Introduction}

A major clinical problem in treating cocaine addiction is the high level of relapse that occurs even after months of drug abstinence (Gawin, 1991). Relapse is triggered by an intense desire or craving for the drug that often results from exposure to environmental stimuli associated with previous drug use. In a rodent model of addiction, rats will press a lever for stimuli previously paired with cocaine self-administration at progressively higher rates as a function of abstinence; larger response rates are observed over longer abstinence periods (Neisewander et al., 2000; Grimm et al., 2001, 2003; Ghitza et al., 2003). As such, Shaham and colleagues proposed that cocaine craving "incubates" over extended drug withdrawal (abstinence), resulting in increased cocaine seeking (Grimm et al., 2001).

An intriguing theme in addiction research is that cocaine exposure and abstinence results in molecular, cellular, and chemical neuroadaptations that may underlie cocaine craving and relapse (Kalivas, 2004; Shaham and Hope, 2005). For example, enhanced responding for cocaine cues after withdrawal is associated with increased levels of BDNF in the accumbens (Acb), ventral tegmental area, and amygdala (Grimm et al., 2003). Furthermore, exposure to cocaine cues after a 1 month abstinence increased ERK (extracellular signal-regulated kinase) phosphorylation in the central amygdala (Lu et al., 2005). Moreover, co-

Received June 22, 2006; revised Feb. 20, 2007; accepted Feb. 24, 2007.

This work was supported by National Institute on Drug Abuse Grants DA14339 (R.M.C.) and DA07244 (J.A.H.).

Correspondence should be addressed to Dr. Regina M. Carelli, Department of Psychology, CB\# 3270, Davie Hall,

The University of North Carolina at Chapel Hill, Chapel Hill, NC 27599-3270. E-mail: rcarelli@unc.edu.

DOI:10.1523/JNEUROSCI.3667-06.2007

Copyright $\odot 2007$ Society for Neuroscience $\quad$ 0270-6474/07/273535-05\$15.00/0 caine seeking after abstinence also results in alterations in the cystine-glutamate transporter in the Acb (Baker et al., 2003).

Electrophysiological recordings in behaving rats allows for an examination of adaptive cellular properties in the brain observed in real time during drug seeking. Acb neurons exhibit changes (increases and/or decreases) in firing rate within seconds of responding for cocaine, and during presentations of cocaineassociated stimuli, that reflect the processing of information about cocaine-directed behavior and environmental factors controlling it (Uzwiak et al., 1997; Carelli, 2000, 2004). Recent studies have shown that Acb activity during drug seeking is dynamic, changing as a function of abstinence. We reported a twofold increase in the number and strength of neurons in the Acb core that encode cocaine-related information during the resumption of cocaine self-administration after 1 month of abstinence (Hollander and Carelli, 2005). West and colleagues reported that the activation of Acb shell neurons by a discriminative stimulus that was previously paired with cocaine availability is maintained after 3-4 weeks of abstinence (Ghitza et al., 2003).

Here, electrophysiological recording procedures were used in behaving rats to determine whether the activation of Acb neurons by cocaine-associated stimuli is enhanced after a 1 month abstinence from cocaine self-administration. Acb activity was examined within a single behavioral session when the cocaine cue was presented unexpectedly to the rat, during responding for the same cue in the absence of the drug (extinction), and during resumption of cocaine self-administration. We report increased activation of Acb core (not shell) neurons by cocaine stimuli after a 1 month abstinence that is maintained regardless of contingency of cue presentation or cocaine availability, reflecting a unique form of cellular plasticity related to cocaine-associated cues. 


\section{Materials and Methods}

Subjects and surgery. Male Sprague Dawley rats (90-120 d old; Harlan, Indianapolis, IN) were used $(n=14)$. Rats had ad libitum access to food (Laboratory Rodent Diet; PMI Nutrition International, Branson, MO) with restricted water $(20-30 \mathrm{ml} / \mathrm{d})$ and prepared for self-administration and extracellular recording in the Acb using established procedures (Carelli et al., 2000). Microwire electrode arrays (eight wires per array; stainless wire with Teflon insulation; $50 \mu \mathrm{m}$ tip diameter per wire) were custom-designed, purchased from a commercial source (NB Labs, Dension TX), and bilaterally implanted 1 week before training in the Acb core or shell (anteroposterior, $+1.7 \mathrm{~mm}$; mediolateral, $1.5 \mathrm{~mm}$; dorsoventral, $6.0-7.5 \mathrm{~mm}$; relative to bregma).

Electrophysiological recordings. Electrophysiology procedures have been described previously (Hollander and Carelli, 2005). Briefly, before the start of each session, subjects were connected to a flexible recording cable attached to a commutator that allowed virtually unrestrained movement within the chamber. Acb activity was recorded differentially between the active and the inactive electrodes from the permanently implanted microwires. On-line isolation and discrimination of neuronal activity was accomplished using a neurophysiological system commercially available (MAP system; SIG board filtering, $250 \mathrm{~Hz}$ to $8 \mathrm{kHz}$; sampling rate, $40 \mathrm{kHz}$; Plexon, Dallas, TX). Individual waveforms corresponding to a single cell were discriminated using template analysis procedures provided by the MAP system and sorted further after each experiment using principal component analysis in Offline Sorter (Plexon). Perievent histograms (PEHs) and stripcharts were constructed using commercially available software (NeuroExplorer; Plexon).

Experimental design. Rats were trained to self-administer cocaine during daily $2 \mathrm{~h}$ sessions. The beginning of the session was signaled by the onset of a cue light above a lever and lever extension. Lever depression (fixed ratio 1 schedule) resulted in intravenous cocaine delivery $(0.33 \mathrm{mg}$ per infusion, $\sim 1 \mathrm{mg} / \mathrm{kg}$ per infusion, $6 \mathrm{~s}$ ) paired with termination of the cue light and simultaneous onset of a tone-house-light-conditioned stimulus (CS; $20 \mathrm{~s}$ ). Training was complete when there was $<10 \%$ variability in press number from the average across 10 consecutive days.

Rats were then randomly divided into two equal groups ( $1 \mathrm{~d}$ vs 1 month); no significant differences were observed between groups in the amount of cocaine consumed during training $\left(t_{(12)}=0.789 ; p>0.05\right)$. Acb core and shell activity was recorded during a single test session conducted the following day ( $1 \mathrm{~d})$ or 1 month later (1 month). During abstinence, rats remained in their home cages without drug. The test session consisted of three phases: (1) CS probes, (2) extinction, and (3) self-administration and CS probes (see Fig. 1a). In phase 1, the cue light above the lever was illuminated, but the lever was not extended. Ten random presentations of the tone-house-light CS ( $5 \mathrm{~s}$ ) were presented by the computer over $15 \mathrm{~min}$. Next, the lever previously associated with cocaine during training was extended into the chamber, and phase 2 (extinction) was initiated. Each lever depression resulted in presentation of the CS (no drug). After $2 \mathrm{~h}$, phase 3 was initiated via a priming infusion of cocaine ( $0.33 \mathrm{mg}$ per infusion; $6 \mathrm{~s}$ ) paired with the CS. Lever presses resulted in a cocaine infusion $(0.33 \mathrm{mg}$ per infusion $)$ and the CS. Additionally, the computer presented CS probes $(15-19 ; 5 \mathrm{~s})$ randomly interspersed between lever presses.

Controls. To examine whether cue-related firing represents a generalized stimulus-evoked discharge independent of associative learning, other rats $(n=7)$ were implanted with electrodes into the Acb core and shell. One week later, Acb cell firing was recorded during 20 probe trials of the tone-house-light stimulus (5 s per probe) randomly presented over $30 \mathrm{~min}$. These cues (identical to those used in self-administration) were never paired with cocaine. Immediately thereafter, each rat received an intraperitoneal injection of cocaine $(20 \mathrm{mg} / \mathrm{kg})$, and $10 \mathrm{~min}$ later, an additional 20 tone-house-light probes were randomly presented $(5 \mathrm{~s}$ per probe over $30 \mathrm{~min}$ ).

Data analysis. The amount of time each rat spent in the quadrant where the cocaine-associated lever had previously been extended during training and the number of approaches toward this quadrant after each CS presentation were recorded (phase 1). Approaches to and time in the quandrant were defined by at least one-half of a body length into and orientation of the rat toward the quadrant. Neural responses to CS probes (phases 1 and 3 and for controls) were characterized by significant changes (increases or decreases) in the firing rate within $5 \mathrm{~s}$ after probe onset (signal) versus $5 \mathrm{~s}$ preceding probe onset (baseline) using $z$-score statistics $(p<0.05) . z$-scores were calculated as $(x-b) / s$, where $x$ is the mean firing rate during the CS, $b$ is the mean firing rate during the baseline period, and $s$ is the SD of the baseline period. During extinction, mean firing rates were calculated for each neuron during lever pressing (signal) versus when rats stopped responding for at least $30 \mathrm{~min}$ (baseline). Signal-to-baseline (S/B) ratios were calculated (dividing signal by baseline) for each cell to determine the strength of the neural correlate during extinction responding. In phase 3 , cell firing was classified into one of four well defined types of phasic neuronal firing patterns within previously defined epochs (Carelli et al., 2000) using $z$-score statistics $(p<0.05)$. Briefly, neurons were classified as type PR if significant increases in activity preceded the reinforced response, as type RFe or RFi if significant increases or decreases in firing occurred immediately after response completion, or as type $\mathrm{PR}+\mathrm{RF}$ if significant dual peak increases were observed before and after the response. The percentages of each type of patterned discharge were determined for each test session and averaged across rats to obtain a mean \pm SEM phasic percentage value; comparisons across groups were accomplished with paired $t$ tests.

\section{Results}

A similar number of electrode placements and recordings were made within the core and shell. However, alterations in cell firing after abstinence were found exclusively in the core, not the shell (see supplemental material, available at www.jneurosci.org, for details and a diagram of electrode placements).

In phase 1 , the 1 month rats demonstrated a preference for the quadrant that previously contained the cocaine lever (Fig. 1b). The 1 month group spent significantly more time in this quadrant $\left(t_{(12)}=4.947 ; p<0.01\right)$ and exhibited significantly more approaches into it $\left(t_{(12)}=6.417 ; p<0.01\right)$ after each CS probe compared with the $1 \mathrm{~d}$ rats.

A total of $170 \mathrm{Acb}$ neurons were recorded in phase 1. Distinct populations of neurons were selectively activated by the CS probes; neurons exhibited either excitatory (Fig. 1c, top) or inhibitory (Fig. 1c, bottom) discharges within seconds of CS probe onset. For the $1 \mathrm{~d}$ group, $7 \%$ of all recorded cells (6 of 90 cells) displayed one of these two types of patterned discharges. In contrast, significantly more Acb neurons (nearly five times) were activated by the CS in the 1 month group compared with the $1 \mathrm{~d}$ rats $\left(28\right.$ of 80 cells; $\left.t_{(11)}=4.419 ; p<0.01\right)$ (Fig. $\left.1 d\right)$. Cells showed an excitatory $(n=24$ cells) or inhibitory $(n=4)$ response relative to CS probe onset in the 1 month group compared with the $1 \mathrm{~d}$ rats ( $n=2$ excitatory; $n=4$ inhibitory). The mean $z$-score for neuronal activity on approach trials (1.58) was over twofold higher than that observed on trials in which rats did not approach the cocaine-associated lever quadrant (0.64).

Next, the lever was extended into the chamber, and each lever press resulted in CS presentation only ("extinction," phase 2). The 1 month group pressed the lever for the CS significantly more times than the $1 \mathrm{~d}$ rats $\left(t_{(12)}=8.819 ; p<0.01\right)$ (Fig. $\left.2 a\right)$. Examples of the activity of two representative neurons recorded during extinction for a $1 \mathrm{~d}$ versus 1 month rat are shown in Figure $2 b$. The $1 \mathrm{~d}$ rat (Fig. $2 b$, top) completed 25 lever presses for the CS during extinction (indicated by the vertical lines above the top $\mathrm{PEH}$ ). The Acb neuron recorded during this phase ( $\mathrm{PEH}$ below the behavioral record) exhibited a mean firing rate during lever pressing of $6.65 \mathrm{~Hz}$ that was reduced as behavior declined later in the phase (mean, $3.55 \mathrm{~Hz}$ ). The bottom portion of Figure $2 b$ shows extinction responding (vertical lines above the $\mathrm{PEH}$ ) for the 1 month rat along with cell firing of another neuron simultaneously recorded. Note that the rat lever pressed at a much higher 
$\mathbf{a}$

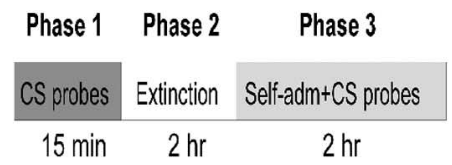

b

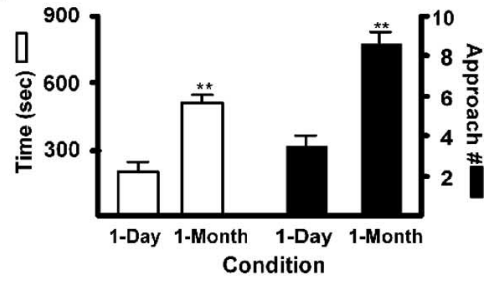

c
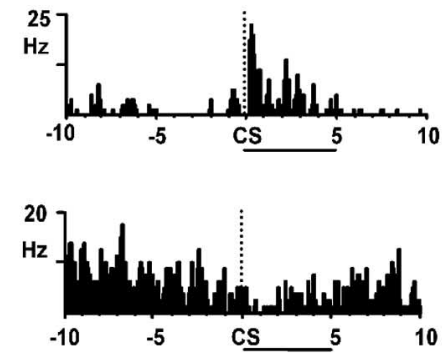

d

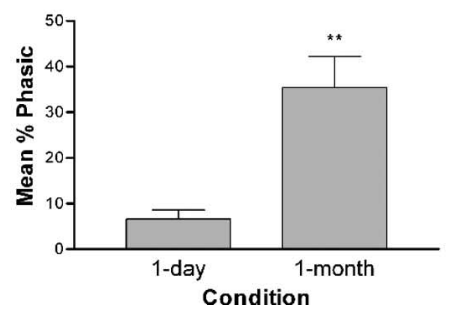

Figure 1. a S Schematic diagram of the test session (see Materials and Methods for details). $\boldsymbol{b}$, CS-induced behavioral activation (time in, and number of approaches to, cocaine-associated lever quadrant) during phase 1. c, PEHs showing examples of individual neurons exhibiting excitatory (top) or inhibitory (bottom) responses relative to $\mathrm{CS}$ onset (indicated by dashed lines in PEHs) in phase 1. (S duration (5 s) is indicated by the horizontal line below each PEH. $\boldsymbol{d}$, Percentage of Acb neurons that were significantly (via z-score analysis) activated by the $C S$ in phase 1 for $1 \mathrm{~d}$ and 1 month rats. ${ }^{* *} p<0.01$.

rate for the CS (122 presses). Moreover, the firing rate during responding was considerably greater for the 1 month rat (mean, $9.28 \mathrm{~Hz}$ ) compared with the $1 \mathrm{~d}$ rat and was reduced later in the phase (mean, $3.87 \mathrm{~Hz}$ ).

Enhanced neural activity during responding for the CS only was observed across the populations of Acb neurons in the 1 month rats. A significant increase in the $\mathrm{S} / \mathrm{B}$ ratio was observed after a 1 month abstinence ( $\mathrm{S} / \mathrm{B}$ ratio, $2.12 \pm 0.28 ; n=80$ cells) compared with the $1 \mathrm{~d}$ group ( $\mathrm{S} / \mathrm{B}$ ratio, $1.35 \pm 0.05 ; n=91$ cells; $t_{(164)}=2.88 ; p<0.01$ ), indicating enhanced neural processing during extinction responding after a 1 month abstinence from cocaine self-administration.

In phase 3 , each lever press again resulted in cocaine infusion paired with the CS as during training, and CS probes were randomly presented interspersed between cocaine-reinforced responses. When rats resumed cocaine self-administration, leverpress response rates did not differ between the two groups $\left(t_{(12)}=\right.$ $0.589 ; p>0.05$ ) (Fig. 3a). Of the 95 cells recorded for the $1 \mathrm{~d}$ group in phase 3, 18 neurons (19\%) exhibited one of the four types of patterned discharges relative to the lever press for intra-

\section{a}

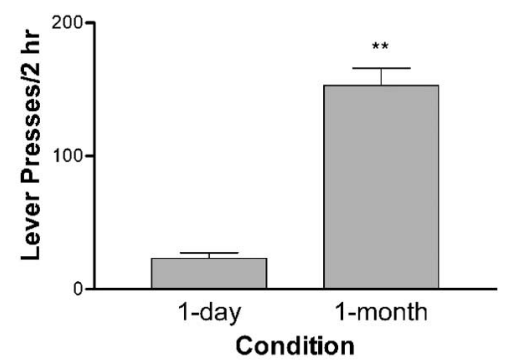

b
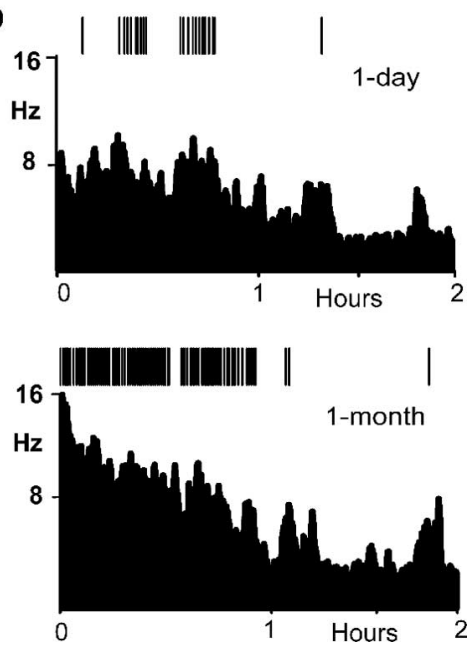

Figure 2. Behavioral responding and Acb cell firing during extinction (phase 2). $\boldsymbol{a}$, Onemonth-abstinent rats exhibited significantly more lever presses for the $C S$ than the $1 \mathrm{~d}$ rats during extinction. ${ }^{* *} p<0.01$. $\boldsymbol{b}$, Examples of representative behavioral response patterns (vertical lines above PEHs) and Acb cell firing in a $1 \mathrm{~d}$ rat (top) versus a 1 month rat (bottom).

venous cocaine (Fig. 3b). As reported previously (Hollander and Carelli, 2005), we found a significant increase in the percentage of Acb neurons showing one of the four types of patterned discharges in the 1 month compared with the $1 \mathrm{~d}$ group $\left(t_{(11)}=\right.$ 3.330; $p<0.01$ ) (Fig. 3c).

The ability of the CS to activate distinct populations of Acb core neurons after a 1 month abstinence was maintained in phase 3. Interestingly, the percentage of neurons activated by the CS was considerably less than that observed in phase 1 (compare Figs. $1 d, 3 d$ ), likely because of the preceding phase when the association between the CS and drug was extinguished. Nevertheless, significantly more neurons $\left(t_{(11)}=3.039 ; p<0.05\right)$ were activated by the CS probes in phase 3 after 1 month of abstinence compared with $1 \mathrm{~d}$ (Fig. $3 d$ ). For $1 \mathrm{~d}$ rats, two cells were excited and two cells were inhibited by the CS. In contrast, for 1 month rats, eight cells were excited and two cells were inhibited by CS presentations. Interestingly, the majority of neurons activated by the CS for both $1 \mathrm{~d}$ and 1 month rats in phase 1 were not the same cells that processed information about the drug-directed behaviors in phase 3 (i.e., types $\mathrm{PR}, \mathrm{RFe}, \mathrm{RFi}, \mathrm{PR}+\mathrm{RF}$ ).

The majority of Acb neurons were not activated by tonehouse-light probes in controls. Specifically, only 2 of 89 cells (2\%) showed a change in firing rate during probe presentation, which remained unchanged ( 2 of 84 cells; $2 \%$ ) after cocaine injection.

\section{Discussion}

The present study reveals that 1 month abstinence from cocaine self-administration causes an increased activation of Acb core neurons to cocaine-associated stimuli. These findings were ob- 
a

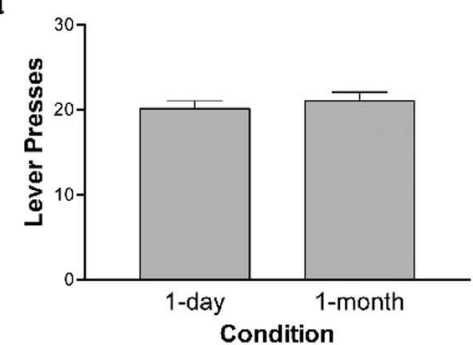

b
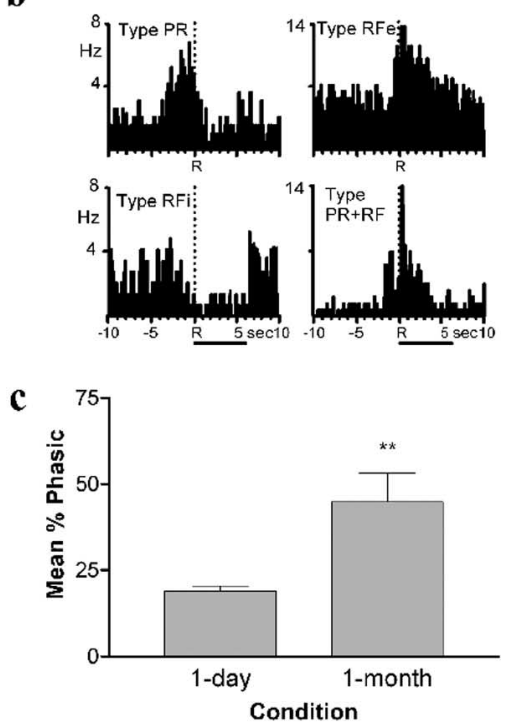

d

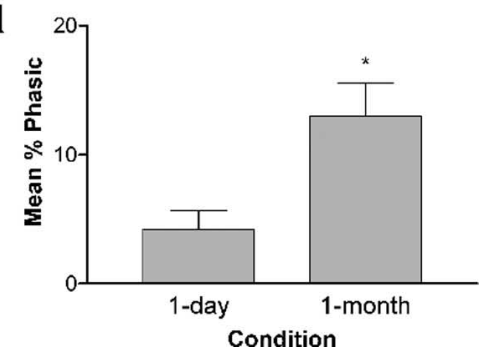

Figure 3. Behavioral responding and Acb cell firing during resumption of cocaine selfadministration (phase 3). $\boldsymbol{a}$, Similar numbers of responses were observed during resumption of self-administration across groups. $\boldsymbol{b}$, Examples of the four types of patterned discharges observed within seconds of the cocaine-reinforced response (indicated by " $\mathrm{R}$ " at the dashed line in PEHs; duration of cocaine delivery indicated by horizontal lines below the bottom PEHs). C, Average percentage of Acb neurons exhibiting one of the four types of patterned discharges significantly increased after a 1 month abstinence. ${ }^{* *} p<0.01$. d, Average percentage of Acb cells that were activated by $C S$ probes was higher in the 1 month compared with the $1 \mathrm{~d}$ group. ${ }^{*} p<0.05$.

served regardless of whether the cocaine cue was presented to the rat or the rat responded for it, in the presence or absence of the drug. Although an ideal control would have been to present the CS and cocaine in a truly random design, because of the relatively long time course of the actions of cocaine (and the inability to ensure that pairings would not have occurred unless very long intervals between the CS and unconditioned stimulus were given), we instead examined whether the same tone-house-light stimulus elicited a generalized stimulus-evoked discharge independent of learning in other rats. Acb neurons exhibited negligible activation by the stimulus when given independent of selfadministration learning, supporting the view that Acb activation by the CS in the present study represents a conditioned activation.
Enhanced behavioral and neural activation by cocaine stimuli after 1 month of abstinence

Presentation of cocaine cues in phase 1 increased drug-seeking behavior in the 1-month-abstinent rats. It is possible that similar findings would be observed beyond 1 month abstinence because Ciccocioppo et al. (2004) reported that conditioned stimuli associated with a single $2 \mathrm{~h}$ cocaine self-administration session elicited drug seeking in rats even after 1 year of abstinence. Moreover, the enhanced cocaine seeking after each CS probe was coincident with the activation of a distinct population of Acb cells. This cellular activation may represent the engagement of specific neural circuits that may trigger drug-seeking behavior and that becomes more responsive after removal of drug access for 1 month.

In human cocaine addicts, exposure to cocaine-associated cues and the resultant drug craving have been well characterized. For example, cocaine addicts who experience cocaine-associated stimuli, rather than neutral or arousing cues, reported higher amounts of subjective craving (Johnson et al., 1998). This increase in cocaine craving was correlated to frontal lobe activation, areas of the brain highly involved in sensory, motor, and emotional processing (Bonson et al., 2002; Kosten et al., 2006). Activation of Acb core neurons by cocaine cues may be driven by the glutamatergic projection from the prefrontal cortex (PFC) and may represent a critical circuit underlying cocaine-seeking behaviors elicited by drug cues (Kalivas, 2004; Kalivas et al., 2005; Rebec and Sun, 2005). Consistent with this, inactivation of the dorsomedial PFC prevents cocaine-associated stimuli from reinstating extinguished drug-seeking behavior (McLaughlin and See, 2003). Thus, it can be postulated that the CS-elicited increase in cocaine seeking and Acb activation observed in the 1 month rats in our study may be dependent on the functional integrity of PFC-Acb circuit. Additionally, structures such as the central amygdala may also play a role in this process (Lu et al., 2005).

Behavioral activation and enhanced neural processing was also observed in 1 month rats during extinction when rats responded for the CS. Our behavioral findings are consistent with work by others showing that rats will lever press for stimuli previously associated with cocaine at increasingly higher rates as the abstinence period is extended (Neisewander et al., 2000; Grimm et al., 2001, 2003; Weiss et al., 2001; Ghitza et al., 2003). The positive correlation of withdrawal (abstinence) duration and cocaine seeking may reflect an "incubation of craving" (Grimm et al., 2001). Our results provide critical insight into the activity of Acb neurons during this process and reveal a possible neural correlate underlying the increase in cocaine seeking.

Enhanced activation of Acb neurons by cocaine-associated cues during resumption of cocaine self-administration Acb neurons exhibit changes (increases and/or decreases) in firing rate within seconds of operant responding for cocaine and during presentations of cocaine-associated stimuli that reflect the encoding of cocaine cues and cocaine-directed behaviors (Carelli, 2000, 2004; Carelli et al., 2000; Uzwiak et al., 1997). This processing of information is dynamic and changes as a function of abstinence. For example, we have reported an increase in the number of Acb neurons that encode cocaine-related information during resumption of self-administration in rats removed from cocaine access for 1 month (Hollander and Carelli, 2005). The present study replicates and extends that report by showing that there is an increase in both the number of phasic cells during cocaine self-administration and in the neural responsiveness to drug-associated stimuli after 1 month of cocaine abstinence. Im- 
portantly, these enhanced neural activations cannot be attributed to an alteration in the pharmacological action of the drug after abstinence, because there were no differences in selfadministration behavior between the $1 \mathrm{~d}$ and 1 month groups. Our findings also indicate that neurons activated by the CS (phase 3) are generally not the same cells that encode cocainedirected behaviors. Collectively, these findings reveal a complex functional organization in the Acb with distinct populations of neurons differentially encoding cocaine self-administration versus cue-induced cocaine seeking after abstinence.

The enhanced activation of neurons by cocaine cues after abstinence was observed exclusively in the core (not shell) of the Acb. This finding is notable given the anatomic and functional differences between these regions (Zahm, 2000). It has been proposed that processing in the Acb core may be predominantly involved in drug seeking, consistent with the view that addiction involves compulsive or automatic behaviors (McFarland and Kalivas, 2001; Ito et al., 2004). Likewise, changes in the core (not shell) have been reported in cue-related dopamine (Ito et al., 2000) and Fos expression (Miller and Marshall, 2005). Similarly, inactivation of the Acb core (not shell) via a GABA agonist mixture prevented conditioned cue-induced reinstatement of cocaine seeking (Fuchs et al., 2004), and the core also plays an essential role in cocaine-primed reinstatement (McFarland and Kalivas, 2001). In contrast, the Acb shell appears to be critical for other types of reward-related processing including, for example, pavlovian-to-instrumental transfer (Corbit et al., 2001) and psychostimulant actions of cocaine (Ito et al., 2004). Interestingly, West and colleagues (Ghitza et al., 2003) showed that neurons in the shell (not core) are activated by a discriminative stimulus previously paired with cocaine availability and remains so even after 3-4 weeks of abstinence. Thus, neural encoding within the core and shell may be differentially modified after cocaine abstinence, reflecting the distinct functional properties of those regions.

\section{Conclusion}

Cocaine exposure and abstinence has been shown to result in molecular, cellular and chemical neuroadaptations in the mesolimbic system believed to play a role in cocaine craving and relapse (Shaham and Hope, 2005; Kalivas, 2004; Robinson and Kolb, 2004). Here, we show increased cocaine seeking coupled to an enhanced activation of Acb neurons in the core during presentation of cocaine-conditioned stimuli after 1 month of abstinence that occurred regardless of drug availability or contingency of cue delivery. As such, our findings reveal novel cellular neuroadaptations in the Acb that are a direct consequence of cocaine abstinence and that are manifested in real time during cocaineseeking behavior.

\section{References}

Baker DA, McFarland K, Lake RW, Shen H, Tang XC, Toda S, Kalivas PW (2003) Neuroadaptations in cystine-glutamate exchange underlie cocaine relapse. Nat Neurosci 6:743-749.

Bonson KR, Grant SJ, Contoreggi CS, Links JM, Metcalfe J, Weyl HL, Kurian V, Ernst M, London ED (2002) Neural systems and cue-induced cocaine craving. Neuropsychopharmacology 26:376-386.

Carelli RM (2000) Activation of accumbens cell firing by stimuli associated with cocaine delivery during self-administration. Synapse 35:238-242.

Carelli RM (2004) Nucleus accumbens cell firing and rapid dopamine signaling during goal-directed behaviors in rats. Neuropharmacology 47:180-198.

Carelli RM, Ijames SG, Crumling AJ (2000) Evidence that separate circuits in the nucleus accumbens encode cocaine versus "natural" (water and food) reward. J Neurosci 20:4255-5266.

Ciccocioppo R, Martin-Fardon R, Weiss F (2004) Stimuli associated with a single cocaine experience elicit long-lasting cocaine-seeking. Nat Neurosci 7:495-496.

Corbit LH, Muir JL, Balleine BW (2001) The role of the nucleus accumbens in instrumental conditioning: evidence of a functional dissociation between accumbens core and shell. J Neurosci 21:3251-3260.

Fuchs RA, Evans KA, Parker MC, See RE (2004) Differential involvement of the core and shell subregions of the nucleus accumbens in conditioned cue-induced reinstatement of cocaine seeking in rats. Psychopharmacology 176:459-465.

Gawin FH (1991) Cocaine addiction: psychology and neurophysiology. Science 251:1580-1586.

Ghitza UE, Fabricatore AT, Prokopenko V, Pawlak AP, West MO (2003) Persistent cue-evoked activity of accumbens neurons after prolonged abstinence from self-administered cocaine. J Neurosci 23:7239-7245.

Grimm JW, Hope BT, Wise RA, Shaham Y (2001) Neuroadaptation. Incubation of craving after withdrawal. Nature 412:141-142.

Grimm JW, Lu L, Hayashi T, Hope BT, Su TP, Shaham Y (2003) Timedependent increases in brain-derived neurotrophic factor protein levels within the mesolimbic dopamine system after withdrawal from cocaine: implications for incubation of cocaine craving. J Neurosci 23:742-747.

Hollander JA, Carelli RM (2005) Abstinence from cocaine selfadministration heightens neural encoding of goal-directed behaviors in the accumbens. Neuropsychopharmacology 30:1464-1474.

Ito R, Dalley JW, Howes SR, Robbins TW, Everitt BJ (2000) Dissociation in conditioned dopamine release in the nucleus accumbens core and shell in response to cocaine cues and during cocaine-seeking behavior in rats. J Neurosci 20:7489-7495.

Ito R, Robbins TW, Everitt BJ (2004) Differential control over cocaineseeking behavior by nucleus accumbens core and shell. Nat Neurosci 7:389-397.

Johnson BA, Chen YR, Schmitz J, Bordnick P, Shafer A (1998) Cue reactivity in cocaine-dependent subjects: effects of cue type and cue modality. Addict Behav 23:7-15.

Kalivas PW (2004) Glutamate systems in cocaine addiction. Curr Opin Pharmacol 4:23-29.

Kalivas PW, Volkow N, Seamans J (2005) Unmanageable motivation in addiction: a pathology in prefrontal-accumbens glutamate transmission. Neuron 45:647-650.

Kosten TR, Scanley BE, Tucker KA, Oliveto A, Prince C, Sinha R, Potenza MN, Skudlarski P, Wexler BE (2006) Cue-induced brain activity changes and relapse in cocaine-dependent subjects. Neuropsychopharmacology 31:644-650.

Lu L, Hope BT, Dempsey J, Liu SY, Bossert JM, Shaham Y (2005) Central amygdala ERK signaling pathway is critical to incubation of cocaine craving. Nat Neurosci 8:212-219.

McFarland K, Kalivas PW (2001) The circuitry mediating cocaine-induced reinstatement of drug-seeking behavior. J Neurosci 21:8655-8663.

McLaughlin J, See RE (2003) Selective inactivation of the dorsomedial prefrontal cortex and the basolateral amygdala attenuates conditioned-cued reinstatement of extinguished cocaine-seeking behavior in rats. Psychopharmacology 168:57-65.

Miller CA, Marshall JF (2005) Altered Fos expression in neural pathways underlying cue-elicited drug seeking in the rat. Eur J Neurosci 21:1385-1393.

Neisewander JL, Baker DA, Fuchs RA, Tran-Nguyen LT, Palmer A, Marshall JF (2000) Fos expression and cocaine-seeking behavior in rats after exposure to a cocaine self-administration environment. J Neurosci 20:798-805.

Rebec GV, Sun W (2005) Neuronal substrates of relapse to cocaine-seeking behavior: role of prefrontal cortex. J Exp Anal Behav 84:653-666.

Robinson TE, Kolb B (2004) Structural plasticity associated with exposure to drugs of abuse. Neuropharmacology 1:33-46.

Shaham Y, Hope BT (2005) The role of neuroadaptations in relapse to drug seeking. Nat Neurosci 8:1437-1439.

Uzwiak AJ, Guyette FX, West MO, Peoples LL (1997) Neurons in accumbens subterritories of the rat: phasic firing time-locked within seconds of intravenous cocaine infusion. Brain Res 767:363-369.

Weiss F, Martin-Fardon R, Ciccocioppo R, Kerr TM, Smith DL, Ben-Shahar O (2001) Enduring resistance to extinction of cocaine-seeking behavior induced by drug-related cues. Neuropsychopharmacology 25:361-371.

Zahm DS (2000) An integrative neuroanatomical perspective on some subcortical substrates of adaptive responding with emphasis on the nucleus accumbens. Neurosci Biobehav Rev 24:85-105. 